\section{Clinical Features and Perforin A91V Gene Analysis in 31 Patients with Macrophage Activation Syndrome and Systemic Juvenile Idiopathic Arthritis in China}

To the Editor:

Macrophage activation syndrome (MAS) is a severe, potentially fatal complication of rheumatoid disease, especially in systemic juvenile idiopathic arthritis (sJIA). MAS has been considered reactive familial hemophagocytic lymphohistiocytosis (FHLH), and the perforin gene mutations have been identified in some patients diagnosed with the FHLH $^{1,2,3}$. Vastert, et $a l^{4}$ have found that the mutation rate of perforin gene A91V was significantly higher in MAS than in sJIA. Perforin deficiency may increase macrophage activation because of an increased production of interferon- $\gamma$ and granulocyte-macrophage colony-stimulating factor associated with persistent lymphocyte activation ${ }^{5,6,7}$. These findings suggest that perforin may play a role in MAS pathogenesis.

A total of 31 patients with MAS were identified from 199 sJIA patients, with a morbidity of $15.57 \%$, in our institute in the last 9 years. We retrospectively reviewed these cases. Gene polymorphisms of perforin A91V (US National Center for Biotechnology Information: single-nucleotide polymorphism rs 35947132 ) were analyzed in 21 of the cases. The clinical features are shown in Table 1. Twenty-five out of 31 patients $(80.64 \%)$ had possible infections for a week to 3 weeks with use of antibiotics prior to developing MAS. The sudden onset of persistent high fever was found in all patients. Progressive hepatosplenomegaly was seen in 29 patients $(93.55 \%)$, and 24 patients $(77.42 \%)$ had elevated aspartate transaminase. Fibrinogen was reduced in 15 patients (48.39\%). All cases had $>1$ series blood cell reduced and pancytopenia occurred in 13 patients (41.9\%). Hemophagocytosis was confirmed in 27 cases $(87.10 \%)$. Unlike data from other reports, the central nervous system dysfunction in our cases was infrequent. A PCR fragment of about 171 base pairs of the perforin A91V was amplified with the forward primer (5'-CAC CCT CTG TGA AAA TGC CCT AC-3') and reverse primer (5'-TTC CAG TCG TTG CGG ATG CTA $\left.C-3^{\prime}\right)^{8}$. All genotypes of these 21 MAS cases were wild-type, in contrast to the findings of Vastert, et $a l^{4}$.

Table 1. Characteristics of 31 cases of macrophage activation syndrome.

\begin{tabular}{lc}
\hline Characteristic & No. Cases $(\%)$ \\
\hline Persistent fever & $31(100)$ \\
Hepatosplenomegaly & $29(93.55)$ \\
Enlarged lymph nodes & $20(64.52)$ \\
Pancytopenia & $13(41.94)$ \\
AST $>60 \mathrm{U} / 1$ & $24(77.42)$ \\
Serum ferritin $>1500 \mathrm{ng} / \mathrm{ml}$ & $27(87.10)$ \\
Fibrinogen $<2.5 \mathrm{~g} / 1$ & $15(48.39)$ \\
Triglyceride $>0.23 \mathrm{mmol} / 1$ & $23(74.19)$ \\
LDH $>159$ U/1 & $27(87.10)$ \\
Creatine kinase $<45 \mathrm{U} / 1$ & $31(100)$ \\
Hemophagocytosis & $27(87.10)$ \\
\hline
\end{tabular}

LDH: lactate dehydrogenase; AST: aspartate transaminase.
MAS is a complex complication of autoimmune disease. The genetic variation of main molecular participation in killing the target cell while treating with intracellular pathogens - the perforin 1 and granzyme B may be involved in the occurrence of MAS in patients with sJIA. In our study, the A91V polymorphic fragments amplified by both primers were sequenced and no mutation was found. Ethnic and regional differences may play a role in the pathogenesis. Further studies and larger cohorts are required to make clear whether the $\mathrm{A} 91 \mathrm{~V}$ is pathogenic in MAS.

ZENG HUASONG, MD, PhD, The First Clinical Medical College of Jinan University; WEI PING, MS; CHEN XIANGYUAN, MS; WEI YANDAN, MS, Department of Allergy, Immunology and Rheumatology, Guangzhou Medical College's affiliated Guangzhou Women and Children's Medical Center, Guangzhou 510120, Guangdong, China. Address correspondence to Dr. Huasong; E-mail: huasongz@gmail.com.

Supported by the Natural Science Foundation of Guangdong Province (1015 1012001000006 ).

\section{REFERENCES}

1. Zhang K, Jordan MB, Marsh RA, Johnson JA, Kissell D, Meller J, et al. Hypomorphic mutations in PRF1, MUNC13-4, and STXBP2 are associated with adult-onset familial HLH. Blood 2011;118:5794-8.

2. Zur Stadt U, Beutel K, Kolberg S, Schneppenheim R, Kabisch H, Janka G, et al. Mutation spectrum in children with primary hemophagocytic lymphohistiocytosis: Molecular and functional analyses of PRF1, UNC13D, STX11, and RAB27A. Hum Mutat 2006;27:62-8.

3. Gholam C, Grigoriadou S, Gilmour KC, Gaspar HB. Familial haemophagocytic lymphohistiocytosis: Advances in the genetic basis, diagnosis and management. Clin Exp Immunol 2011;163:271-83.

4. Vastert SJ, van Wijk R, D’Urbano LE, de Vooght KM, de Jager W, Ravelli A, et al. Mutations in the perforin gene can be linked to macrophage activation syndrome in patients with systemic onset juvenile idiopathic arthritis. Rheumatology 2010;49:441-9.

5. Grom AA, Mellins ED. Macrophage activation syndrome: Advances towards understanding pathogenesis. Curr Opin Rheumatol 2010;22:561-6.

6. Pintaric M, Gerner W, Saalmüller A. Synergistic effects of IL-2, IL-12 and IL-18 on cytolytic activity, perforin expression and IFN-gamma production of porcine natural killer cells. Vet Immunol Immunopathol 2008;121:68-82.

7. Voskoboinik I, Dunstone MA, Baran K, Whisstock JC, Trapani JA. Perforin: structure, function, and role in human immunopathology. Immunol Rev 2010;235:35-54.

8. Mehta PA, Davies SM, Kumar A, Devidas M. Perforin polymorphism A91V and susceptibility to B-precursor childhood acute lymphoblastic leukemia: A report from the Children's Oncology Group. Leukemia 2006;20:1539-41.

J Rheumatol 2013;40:7; doi:10.3899/jrheum.121040 